\title{
Exhaled Eicosanoids following Bronchial Aspirin Challenge in Asthma Patients with and without Aspirin Hypersensitivity: The Pilot Study
}

\author{
L. Mastalerz, M. Sanak, J. Kumik, A. Gawlewicz-Mroczka, N. Celejewska-Wójcik, \\ A. Ćmiel, and A. Szczeklik
}

Department of Medicine, Medical College, Jagiellonian University, ul. Skawińska 8, 31-066 Kraków, Poland

Correspondence should be addressed to A. Szczeklik, mmszczek@cyf-kr.edu.pl

Received 13 July 2011; Accepted 30 September 2011

Academic Editor: A. P. Sampson

Copyright (C) 2012 L. Mastalerz et al. This is an open access article distributed under the Creative Commons Attribution License, which permits unrestricted use, distribution, and reproduction in any medium, provided the original work is properly cited.

\begin{abstract}
Background. Special regulatory role of eicosanoids has been postulated in aspirin-induced asthma. Objective. To investigate effects of aspirin on exhaled breath condensate (EBC) levels of eicosanoids in patients with asthma. Methods. We determined EBC eicosanoid concentrations using gas chromatography/mass spectrometry (GC-MS) and high-performance liquid chromatography/mass spectrometry (HPLC-MS ${ }^{2}$ ) or both. Determinations were performed at baseline and following bronchial aspirin challenge, in two well-defined phenotypes of asthma: aspirin-sensitive and aspirin-tolerant patients. Results. Aspirin precipitated bronchial reactions in all aspirin-sensitive, but in none of aspirin-tolerant patients (ATAs). At baseline, eicosanoids profile did not differ between both asthma groups except for lipoxygenation products: 5- and 15-hydroxyeicosatetraenoic acid (5-, 15-HETE) which were higher in aspirin-induced asthma (AIA) than inaspirin-tolerant subjects. Following aspirin challenge the total levels of cysteinyl-leukotrienes (cys-LTs) remained unchanged in both groups. The dose of aspirin had an effect on magnitude of the response of the exhaled cys-LTs and prostanoids levels only in AIA subjects. Conclusion. The high baseline eicosanoid profiling of lipoxygenation products 5- and 15-HETE in EBC makes it possible to detect alterations in aspirin-sensitive asthma. Cysteinylleukotrienes, and eoxins levels in EBC after bronchial aspirin administration in stable asthma patients cannot be used as a reliable diagnostic index for aspirin hypersensitivity.
\end{abstract}

\section{Introduction}

Exhaled breath condensate (EBC) is a simple, noninvasive technique for monitoring airway inflammation. The measurement of eicosanoids in the expired breath condensate has proven to be a useful noninvasive method for the assessment and monitoring of airway inflammation in inflammatory diseases such as asthma and other pulmonary diseases $[1,2]$.

Eicosanoids, including prostaglandins (PGs), thromboxane $\mathrm{A}_{2}\left(\mathrm{TXA}_{2}\right)$, and leukotrienes (LTs), are lipid mediators involved in the pathogenesis of asthma. TXA 2 is rapidly converted to thromboxane $\mathrm{B}_{2}\left(\mathrm{TXB}_{2}\right)$, a chemically stable metabolite. Thus, thromboxane synthesis in biological tissues has been monitored by measuring $\mathrm{TXB}_{2}[1,2]$. Eoxins $\mathrm{C}_{4}, \mathrm{D}_{4}$, and $\mathrm{E}_{4}\left(\mathrm{EXC}_{4}, \mathrm{EXD}_{4}, \mathrm{EXE}_{4}\right)$ are 15-lipoxygenase (15-LO) analogues of cysteinyl leukotrienes (cys-LTs). Eoxins were metabolized to eoxin $\mathrm{E}_{4}$ and detectable in EBC [3].
Another group of lipoxygenation products is 5-, 12- and 15-hydroxyeicosatetraenoic acid (5-HETE, 12-HETE, 15HETE). Isoeicosanoids or isoprostanes are prostaglandinlike compounds produced by nonenzymatic lipid peroxidation of arachidonic acid. The 8-isoprostane is the best biomarker of oxidative stress and lipid peroxidation [1-3].

A special regulatory role of eicosanoids was postulated in aspirin-induced asthma (AIA) [4-6]. AIA is characterized by nasal polyps, persistent asthma, and aspirin hypersensitivity [4]. These nasal/sinus and bronchial syndromes with aspirin hypersensitivity have been named aspirin-exacerbated respiratory disease (AERD). Indeed, in this distinct asthma phenotype [7], observations have accumulated pointing to (1) overproduction of cysteinyl-leukotrienes (cys-LTs), which are potent proinflamatory mediators and bronchoconstrictors $[4,6,8],(2)$ upon ingestion of aspirin, cys-LTs are further released in rising amounts probably from eosinophils 
TABLE 1: Clinical characteristics of the patients.

\begin{tabular}{|c|c|c|c|}
\hline & $\operatorname{AIA}(n=21)$ & $\operatorname{ATA}(n=23)$ & $P$ \\
\hline Age $(y)$ & $\begin{array}{l}44.9 \pm 13.96 \\
39(34 \div 58)\end{array}$ & $\begin{array}{c}39.8 \pm 8.71 \\
39(32 \div 45)\end{array}$ & N.S. $(0.33)$ \\
\hline Female/Male & $10 / 11$ & $15 / 8$ & N.S. (0.36) \\
\hline Duration of asthma $(y)$ & $\begin{array}{c}12.36 \pm 8.07 \\
13(6 \div 18)\end{array}$ & $\begin{array}{c}10.26 \pm 11.33 \\
7(1 \div 17)\end{array}$ & N.S. (0.19) \\
\hline Inhaled steroids yes/no & $18 / 3$ & $22 / 1$ & N.S. $(0.34)$ \\
\hline Inhaled steroids $(\mu \mathrm{g} / \mathrm{d})$ flutikason & $\begin{array}{c}616.57 \pm 425.83 \\
500(500 \div 1000)\end{array}$ & $\begin{array}{c}726.09 \pm 667.06 \\
500(250 \div 1000)\end{array}$ & N.S. $(0.93)$ \\
\hline $\begin{array}{l}\mathrm{FEV}_{1} \text { baseline (\% predicted) } \\
\text { placebo/aspirin day }\end{array}$ & $\begin{array}{c}89.87 \pm 10.83 \\
90.01(83.45 \div 95.29)\end{array}$ & $\begin{array}{c}91.45 \pm 11.47 \\
91.3(84.81 \div 102.1)\end{array}$ & N.S. $(0.57)$ \\
\hline Total IgE (IU/mL) & $\begin{array}{c}115.06 \pm 111.41 \\
76.3(42.8 \div 158)\end{array}$ & $\begin{array}{c}162.86 \pm 177.82 \\
66.8(24.1 \div 179)\end{array}$ & N.S. $(0.78)$ \\
\hline $\begin{array}{l}\text { Skin prick test }(n) \\
\text { positive/negative }\end{array}$ & $10 / 9$ & $15 / 8$ & N.S. $(0.53)$ \\
\hline Blood eosinophil count & $\begin{array}{c}472.67 \pm 295.39^{*} \\
424(324 \div 513)\end{array}$ & $\begin{array}{c}248.22 \pm 173.32^{*} \\
232(119 \div 309)\end{array}$ & 0.003 \\
\hline
\end{tabular}

Values are expressed as mean \pm SD, and median ( $25 \%$ and $75 \%$ percentiles). AIA: aspirin-induced asthma.

ATA: aspirin-tolerant asthma.

and mast cells and this is accompanied by worsening of asthmatics symptoms $[9,10],(3)$ depressed prostaglandin $\mathrm{E}_{2}$ $\left(\mathrm{PGE}_{2}\right)$ production by peripheral blood cells [11], nasal polyps [12, 13], bronchial fibroblasts [14], diminished $\mathrm{EP}_{2}$ receptor on the inflammatory cells, and association with $\mathrm{EP}_{2}$ gene polymorphism [15, 16], (4) aspirin-precipitated asthmatic attacks are not associated with changes in the systemic prostaglandin $\mathrm{E}_{2}$ production, it might stem from the release of $\mathrm{PGE}_{2}$ from inflammatory cells during the clinical reactions to aspirin [17], (5) both prostaglandin $\mathrm{D}_{2}\left(\mathrm{PGD}_{2}\right)$ and its metabolite increase after aspirin-induced bronchoconstriction suggesting that this reaction is associated with mast cell activation [18-20].

Aspirin-induced changes in the levels of eicosanoids, such as prostaglandins (PGs) and cys-LTs, have been examined in various biologic samples, such as plasma [19, 20], saliva [19], induced sputum [19, 21], nasal washing fluid [22, 23], bronchoalveolar lavage [24], and urine [9, 10, 17, 25]. So far, there are few published articles on eicosanoids in EBC in aspirin-induced-asthma patients [26-28]. These studies concentrated on baseline levels of eicosanoids in EBC independent of any steroid therapy used. Interestingly, one of these studies reported prostaglandins $\left(\mathrm{PGE}_{2}, \mathrm{PGF}_{2} \alpha\right.$, $9 \alpha 11 \beta \mathrm{PGF}_{2}$ ) and cys-LTs levels in breath condensates in asthmatic patients after oral aspirin challenge [28]. In the present study, we focus on exhaled breath condensate concentrations of eicosanoids following bronchial aspirin challenge, local administration of aspirin in aspirin-induced asthma patients.

The aim of this study was to evaluate the changes in wide eicosanoid spectrum concentrations in EBC during asthmatic response following aspirin inhalation. We hypothesized that the profile of eicosanoids in EBC after local aspirin administrated is markedly different in aspirin-induced asthma patients as compared to asthmatics who tolerate aspirin well. These results were validated by specific analytical techniques, such as gas chromatography/mass spectrometry (GC-MS) or by high-performance liquid chromatography/mass spectrometry (HPLC-MS).

\section{Material and Methods}

2.1. Subjects. The study population consisted of 21 asthmatic patients sensitive to aspirin (AIA) and 23 asthmatics who tolerated aspirin well (ATA). The diagnosis of asthma was established according to GINA 2008 update. The patients' characteristics are presented in Table 1.

The diagnosis of aspirin intolerance was confirmed by oral aspirin provocation tests, performed during 36 months preceding the study. All ATA patients occasionally used aspirin without any adverse reactions. The patients had stable asthma and their baseline $\mathrm{FEV}_{1}$ was $>70 \%$ of the predicted value on the study day. None had experienced an exacerbation or a respiratory tract infection in the 6 weeks preceding the study. Nearly $70 \%$ of ATA patients had intermittent asthma, $13 \%$ mild persistent asthma, and 17\% moderate persistent. In AIA group, $67 \%$ of patients had intermittent asthma, 19\% mild persistent asthma, and 14\% moderate persistent asthma. According to Asthma Control Test 33\% of AIA patients and $26 \%$ of ATA patients had controlled asthma, and $33 \%$ and $48 \%$ had partly controlled asthma, $33 \%$ and $26 \%$ had uncontrolled asthma, respectively. In ATA group, 2 patients were current smokers and 3 exsmokers. There were 7 exsmokers and no current smokers in AIA group. The average level of $\mathrm{FEV}_{1}$ and $\mathrm{FEV}_{1} / \mathrm{FVC}$ in AIA patients was $89.9 \%$ and $73.2 \%$, in ATA patients was $91.5 \%$ and $78.5 \%$, respectively.

The subjects were instructed to withhold medications that decrease bronchial responsiveness prior to aspirin challenge. Short-acting $\beta_{2}$-agonists were not used 8 hours before the challenge. Long-acting $\beta_{2}$-agonists and theophylline were 
TABLE 2: Eicosanoids values at baseline and following aspirin challenge in AIA and ATA patients. Results of eicosanoids were recalculated as parts per million (ppm) of palmitic acid (PA).

\begin{tabular}{|c|c|c|c|c|c|}
\hline & \multicolumn{2}{|c|}{$\operatorname{AIA}(n=21)$} & \multicolumn{2}{|c|}{$\operatorname{ATA}(n=23)$} & \multirow{2}{*}{$\begin{array}{c}P \text { (ANOVA) } \\
\text { After the challenge }\end{array}$} \\
\hline & Baseline & Challenge & Baseline & Challenge & \\
\hline $\mathrm{PGD}_{2}$ & $5.22 \pm 5.93$ & $4.85 \pm 3.92$ & $4.71 \pm 3.38$ & $4.36 \pm 2.83$ & \\
\hline (parts/million of PA) & 3.18 & 3.69 & 4.27 & 3.85 & 0.54 \\
\hline GC/MS & $(1.35 \div 5.85)$ & $(1.56 \div 6.99)$ & $(2.02 \div 6.37)$ & $(1.78 \div 6.31)$ & \\
\hline $\mathrm{PGD}_{2}$ & $4.65 \pm 3.14$ & $3.76 \pm 2.91$ & $3.85 \pm 2.06$ & $3.72 \pm 1.58$ & \\
\hline (parts/million of PA) & 3.56 & 3.00 & 2.99 & 3.33 & Unable \\
\hline HPLC/MS/MS & $(1.93 \div 7.02)$ & $(1.81 \div 5.66)$ & $(2.11 \div 5.38)$ & $(2.47 \div 4.83)$ & \\
\hline $9 \alpha 11 \beta \mathrm{PGF}_{2}$ & $2.20 \pm 2.01$ & $2.21 \pm 2.02$ & $0.73 \pm 0.39$ & $0.79 \pm 0.44$ & \\
\hline (parts/million of PA) & 1.24 & 1.30 & 0.61 & 0.75 & Unable \\
\hline GC/MS & $(0.27 \div 4.34)$ & $(0.28 \div 3.90)$ & $(0.45 \div 1.05)$ & $(0.45 \div 1.02)$ & \\
\hline PGF2 $\alpha$ & $1.67 \pm 1.59$ & $1.41 \pm 1.38$ & $1.14 \pm 1.26$ & $0.99 \pm 1.18$ & \\
\hline (parts/million of PA) & 0.97 & 0.86 & 0.64 & 0.49 & 0.82 \\
\hline GC/MS & $(0.26 \div 2.71)$ & $(0.42 \div 2.16)$ & $(0.43 \div 1.16)$ & $(0.38 \div 1.38)$ & \\
\hline 6-keto-PGF1 $\alpha$ & $30.23 \pm 18.50$ & $29.27 \pm 16.26$ & $27.98 \pm 24.71$ & $29.64 \pm 27.20$ & \\
\hline (parts/million of PA) & 32.15 & 32.96 & 16.80 & 16.12 & 0.74 \\
\hline GC/MS & $(11.76 \div 45.67)$ & $(17.09 \div 37.44)$ & $(12.20 \div 39.60)$ & $(13.82 \div 41.81)$ & \\
\hline 6-keto-PGF1 $\alpha$ & $30.51 \pm 19.45$ & $28.92 \pm 16.65$ & $26.79 \pm 23.71$ & $28.77 \pm 25.48$ & \\
\hline (parts/million of PA) & 32.08 & 32.51 & 15.60 & 18.15 & 0.11 \\
\hline HPLC/MS/MS & $(11.13 \div 44.05)$ & $(15.78 \div 38.12)$ & $(11.91 \div 35.68)$ & $(13.20 \div 28.65)$ & \\
\hline 11-dehydro TXB2 & $19.34 \pm 10.77$ & $20.16 \pm 11.23$ & $16.43 \pm 8.14$ & $17.72 \pm 9.79$ & \\
\hline (parts/million of PA) & 21.67 & 21.40 & 15.10 & 15.55 & 0.82 \\
\hline GC/MS & $(12.18 \div 28.14)$ & $(12.25 \div 26.23)$ & $(9.77 \div 21.96)$ & $(11.74 \div 17.17)$ & \\
\hline 11-dehydro TXB2 & $18.86 \pm 10.82$ & $20.50 \pm 11.95$ & $16.15 \pm 8.20$ & $16.87 \pm 8.81$ & \\
\hline (parts/million of PA) & 19.69 & 21.48 & 14.49 & 14.65 & 0.66 \\
\hline HPLC/MS/MS & $(9.18 \div 27.50)$ & $(11.26 \div 25.83)$ & $(9.37 \div 22.04)$ & $(10.97 \div 19.78)$ & \\
\hline $\mathrm{LTC}_{4}$ & $14.51 \pm 15.87$ & $15.57 \pm 36.94^{*}$ & $9.76 \pm 14.06$ & $4.16 \pm 3.08^{*}$ & \\
\hline (parts/million of PA) & 9.22 & 6.61 & 5.40 & 3.24 & $0.003^{*}$ \\
\hline HPLC/MS/MS & $(2.18 \div 22.31)$ & $(2.08 \div 11.50)$ & $(2.79 \div 10.84)$ & $(1.65 \div 5.84)$ & \\
\hline $\mathrm{LTD}_{4}$ & $3.11 \pm 2.80$ & $2.68 \pm 2.21$ & $3.31 \pm 2.59$ & $2.57 \pm 1.55$ & \\
\hline (parts/million of PA) & 2.18 & 2.25 & 2.68 & 2.16 & 0.67 \\
\hline HPLC/MS/MS & $(0.83 \div 4.41)$ & $(1.22 \div 3.53)$ & $(1.60 \div 4.41)$ & $(1.42 \div 3.58)$ & \\
\hline $\mathrm{LTE}_{4}$ & $6.14 \pm 3.79$ & $11.96 \pm 19.51^{*}$ & $5.45 \pm 2.83$ & $6.47 \pm 2.57^{*}$ & \\
\hline (parts/million of PA) & 5.19 & 6.38 & 4.88 & 6.54 & $0.03^{*}$ \\
\hline HPLC/MS/MS & $(3.23 \div 8.50)$ & $(4.95 \div 14.57)$ & $(3.10 \div 7.76)$ & $(4.42 \div 7.59)$ & \\
\hline Total cysLTs & $23.40 \pm 19.22$ & $30.54 \pm 56.90$ & $18.51 \pm 15.42$ & $13.20 \pm 5.41$ & \\
\hline (parts/million of PA) & 19.74 & 15.70 & 14.14 & 12.94 & 0.33 \\
\hline HPLC/MS/MS & $(6.67 \div 31.22)$ & $(6.68 \div 29.20)$ & $(10.95 \div 21.25)$ & $(8.17 \div 18.75)$ & \\
\hline $\mathrm{LTB}_{4}$ & $154.84 \pm 187.68$ & $73.88 \pm 77.38^{*}$ & $101.37 \pm 163.65$ & $70.69 \pm 73.37$ & \\
\hline (parts/million of PA) & 70.28 & 50.49 & 49.72 & 32.29 & $0.03^{*}$ \\
\hline HPLC/MS/MS & $(24.57 \div 168.26)$ & $(28.71 \div 86.75)$ & $(19.05 \div 126.82)$ & $(19.01 \div 91.91)$ & \\
\hline 5 HETE & $23.95 \pm 39.08^{*}$ & $11.21 \pm 11.26$ & $6.08 \pm 3.83^{*}$ & $5.41 \pm 4.41$ & \\
\hline (parts/million of PA) & 9.98 & 6.78 & 4.53 & 4.22 & Unable \\
\hline HPLC/MS/MS & $(4.71 \div 17.95)$ & $(2.92 \div 16.24)$ & $(3.82 \div 8.32)$ & $(2.63 \div 6.68)$ & \\
\hline 12 HETE & $23.58 \pm 25.35$ & $14.58 \pm 17.15$ & $9.22 \pm 6.48$ & $7.17 \pm 9.85^{*}$ & \\
\hline (parts/million of PA) & 12.49 & 6.62 & 7.93 & 5.23 & Unable \\
\hline HPLC/MS/MS & $(4.27 \div 32.00)$ & $(4.31 \div 15.53)$ & $(4.11 \div 12.84)$ & $(3.87 \div 6.26)$ & \\
\hline
\end{tabular}


TABle 2: Continued.

\begin{tabular}{lccccc}
\hline & \multicolumn{2}{c}{ AIA $(n=21)$} & \multicolumn{2}{c}{ ATA $(n=23)$} & Ch (ANOVA) \\
& Baseline & Challenge & Baseline & Challenge & After the challenge \\
\hline 15 HETE & $22.65 \pm 24.08^{*}$ & $14.84 \pm 9.22$ & $7.73 \pm 5.52 *$ & $10.47 \pm 17.07$ \\
(parts/million of PA) & 15.48 & 12.80 & 6.72 & 6.13 & 0.23 \\
HPLC/MS/MS & $(7.80 \div 24.57)$ & $(8.35 \div 15.39)$ & $(3.17 \div 11.75)$ & $(4.62 \div 11.25)$ \\
\hline EXC 4 & $2.72 \pm 2.45$ & $2.99 \pm 3.08$ & $2.00 \pm 1.67$ & $1.99 \pm 1.69$ \\
(parts/million of PA) & 2.09 & 2.59 & 1.41 & 1.61 & $(0.68 \div 2.38)$ \\
HPLC/MS/MS & $(1.17 \div 3.42)$ & $(0.65 \div 4.32)$ & $(1.09 \div 2.38)$ & $2.87 \pm 2.73$ \\
\hline EXD 4 & $2.98 \pm 2.21$ & $2.77 \pm 2.52$ & $3.03 \pm 2.50$ & 2.22 & 0.79 \\
(parts/million of PA) & 2.12 & 1.97 & 2.57 & $(0.97 \div 4.46)$ \\
HPLC/MS/MS & $(0.82 \div 4.57)$ & $(1.12 \div 4.13)$ & $8.09 \pm 9.65$ & $5.65 \pm 5.28$ \\
\hline EXE 4 & $7.86 \pm 5.11$ & $8.48 \pm 16.15$ & 3.79 & 3.65 \\
(parts/million of PA) & 7.06 & 3.82 & $(2.67 \div 12.23)$ & $(2.33 \div 8.97)$ \\
HPLC/MS/MS & $(4.00 \div 11.43)$ & $(2.89 \div 7.03)$ & $0.72 \pm 0.26$ & $0.82 \pm 0.32$ \\
\hline 8 -iso-PGF2 $\alpha$ & $0.73 \pm 0.40$ & $0.79 \pm 0.38$ & 0.67 & 0.73 \\
(parts/million of PA) & 0.68 & 0.76 & $(0.58 \div 0.83)$ & $(0.55 \div 1.08)$ \\
GC/MS & $(0.40 \div 1.04)$ & $(0.54 \div 1.11)$ & & 0.95 \\
\hline
\end{tabular}

Median (25\% and $75 \%$ percentiles).

AIA: aspirin-induced asthma. ATA: aspirin-tolerant asthma. PA: palmitic acid.

${ }^{*} P$-values $<0.05$; AIA versus ATA at baseline or after the challenge.

$P$-values:

* AIA versus ATA at baseline.

* baseline versus challenge in AIA.

* baseline versus challenge in ATA.

withdrawn for 24 hours. Short-acting antihistamines and cromones were stopped 5 days before the challenge. Inhaled steroids were allowed at a dose $\leq 2000 \mu$ g budesonide per day. None of the patients were treated with systemic corticosteroids or leukotriene modifying drugs.

Baseline and following bronchial aspirin challenge, exhaled breath condensate eicosanoids (see Table 2) levels were measured in all subjects. The patients gave informed consent and the study was approved by the University Ethics Committee.

2.2. Study Design. The single-blind, placebo-controlled bronchial challenge test with aspirin was carried out during one day in all study patients [29]. The test began with the inhalation of 7 breaths of placebo (saline). $\mathrm{FEV}_{1}$ was measured at 10 and 20 minutes after placebo inhalation. The postsaline $\mathrm{FEV}_{1}$ obtained at 20 minutes was used as "postsaline baseline" value.

The consecutive doses of lysine-aspirin were inhaled every 30 minutes by increasing the concentration of lysineaspirin and by changing the number of breaths (increasing doses of $0.18,0.36,0.90,2.34,7.20,16.2,39.60,115.20 \mathrm{mg}$, at 0.5 hour intervals, up to the cumulative dose of $181.98 \mathrm{mg}$ ). $\mathrm{FEV}_{1}$ was measured at 10,20, and 30 minutes after each dose. The challenge procedure with aspirin was interrupted, if a bronchospastic reaction occurred ( $\mathrm{FEV}_{1}$ dropped $\geq 20 \%$ ), or if the maximum cumulative dose of aspirin was reached. The cumulative dose of aspirin causing a $20 \%$ fall in $\mathrm{FEV}_{1}$ was calculated and recorded as $\mathrm{PD}_{20}$ (provocation dose of aspirin). $\mathrm{FEV}_{1}$ and extrabronchial symptoms were recorded at baseline, before the challenge tests, and then every 30 minutes until 6 hours after the last dose of aspirin.

In patients with positive bronchial aspirin challenge (AIA), exhaled breath condensate samples were collected for wide eicosanoid spectrum (see Table 2) estimations at baseline and at the time of appearance of the bronchial symptoms (time 0). In ATA patients, whose aspirin challenge was negative, exhaled breath condensate samples were collected at baseline and 0.5 hours after the last aspirin dose, that is, when the cumulative doses of $181.98 \mathrm{mg}$ was reached (time $0)$.

2.3. Lung Function. Pulmonary function tests were performed on a flow-integrating computerized pneumotachograph (Pneumoscreen, E. Jaeger, Germany).

2.4. Exhaled Breath Condensate (EBC). EBC was collected according to ATS/ERS [1] using ECO Screen instrument of Jaeger (GmbH Hoechberg, Germany). Following tidal breathing for 15-20 min, 1-2 mL of clear fluid was collected and immediately deeply frozen.

2.5. Biochemical Assays. Exhaled breath condensate concentration of eicosanoids was measured by gas chromatography/mass spectrometry (GC-MS) and by high-performance liquid chromatography/tandem mass spectrometry (HPLC$\mathrm{MS}^{2}$ ) or both; see Table 2. Results of eicosanoids were recalculated as parts per million (ppm) of palmitic acid (PA) 
or expressed as picograms per milliliter $(\mathrm{pg} / \mathrm{mL})$. Detection limits for eicosanoids measurements were between $0.17 \mathrm{pg} / \mathrm{mL}$ for 12 -HETE and $0.89 \mathrm{pg} / \mathrm{mL}$ for $\mathrm{PGD}_{2}$. Intraassay coefficients of variance were less than $10 \%$ and interassay coefficients of variance were less than $15 \%$. Accuracy of measurements were better than $98.7 \%$. Detailed analytical procedure and deuterated standards used were described elsewhere [3,27].

2.6. Statistical Analysis. Summary statistics were expressed as mean $(\mathrm{M})$, standard deviation (SD), median (Me), and $25 \%$ and $75 \%$ percentiles. General linear model (GLM) including repeated measures analysis of variance, which takes into account the fact that the outcome measurements are repeated over time within subject was used for multiple comparisons. Logarithmic transformation was used when needed as variance stabilizing transformation. Correlation between variables was estimated with the Spearman rank order correlations. A $P$-value $\leq 0.05$ was considered statistically significant.

\section{Results}

3.1. Clinical Reactions. There was no statistical difference in the clinical characteristics between patients with aspirininduced asthma (positive aspirin challenge test), and those who tolerated aspirin well (negative aspirin challenge test) except for blood eosinophil count, Table 1. None of the patients developed symptoms after administration of placebo. In aspirin-sensitive asthmatics, bronchial reactions developed after $0.18 \mathrm{mg}$ in 1 subject, after $0.36 \mathrm{mg}$ in 2 subjects, after $0.9 \mathrm{mg}$ in 3 subjects, following $2.34 \mathrm{mg}$ in 2 , after $7.2 \mathrm{mg}$ in 5 subjects, after $16.2 \mathrm{mg}$ in 4 subjects, following $39.6 \mathrm{mg}$ in 2 subjects, and after $115.2 \mathrm{mg}$ in 2 subjects. The mean cumulative dose of aspirin was $32.108 \mathrm{mg}$. All the symptoms were relieved by short-acting $\beta_{2}$-agonists. None of ATA patients developed any clinical symptoms following aspirin challenges.

\subsubsection{Cyclooxygenase Products}

Exhaled Breath Condensate Concentration of Prostanoids. At baseline (Table 2), exhaled breath condensate levels of $\mathrm{PGD}_{2}$, such as its metabolite $9 \alpha 11 \beta \mathrm{PGF}_{2}$, and $\mathrm{PGF}_{2} \alpha$, 6-keto- $\mathrm{PGF}_{1} \alpha$ and nonenzymatic isomer (8-iso-PGF 2 ), did not differ significantly between the study groups. Statistically, results did not differ dependently on the methods used (HPLC/MS/MS or GC/MS) for marking eicosanoids and results shown (ppm of PA or $\mathrm{pg} / \mathrm{mL}$ ).

Following aspirin administration, no significant differences in EBC levels of $\mathrm{PGD}_{2}$ measured by HPLC/MS/MS (results were recalculated as $\mathrm{ppm}$ of $\mathrm{PA}$ ) and $9 \alpha 11 \beta \mathrm{PGF}_{2}$ measured by GC/MS (results were expressed as ppm of PA) were found in AIA $(P=0.13$ and $P=0.82$, resp. $)$ and in ATA subjects $(P=0.69$ and $P=0.43$, resp.). The same marked result (no differences) was observed when $\mathrm{PGD}_{2}$ was measured by GC-MS.
In cases when 11-dehydro $\mathrm{TXB}_{2}$ resulted as $\mathrm{ppm}$ of $\mathrm{PA}$, at baseline (Table 2) and following aspirin challenge (ANOVA, $P=0.66)$ exhaled breath condensate level of 11-dehydro $\mathrm{TXB}_{2}$ did not differ significantly between the study groups.

Negative correlation was founded between provocation doses of aspirin and exhaled $\mathrm{PGD}_{2}$ and its $9 \alpha 11 \beta \mathrm{PGF}_{2}$, $\mathrm{PGF}_{2} \alpha, 6$-keto- $\mathrm{PGF}_{1} \alpha$, and 11-dehydro $\mathrm{TXB}_{2}$ levels only in AIA patients.

\subsubsection{Lipoxygenation Products}

Exhaled Breath Condensate Concentration of CysteinylLeukotriene (Cys-LTs). At baseline (Table 2), exhaled breath condensate levels of leukotrienes $\mathrm{C}_{4}, \mathrm{D}_{4}$, and $\mathrm{E}_{4}$ did not differ significantly between the AIA and ATA groups $(P=0.43$, $P=0.22, P=0.79$, resp.).

In both study groups, following aspirin challenge, $\mathrm{EBC}$ level of $\mathrm{LTC}_{4}$ decreased significantly (ANOVA, $P=0.003$ ). No significant differences in EBC levels of $\mathrm{LTD}_{4}$ was found in AIA and in ATA group patients (ANOVA, $P=0.67$ ), where the level remained unchanged and at a constant. Exhaled $\mathrm{LTE}_{4}$ after aspirin challenge increased significantly in both study groups (ANOVA, $P=0.03$ ). However, total level of cys-LTs (the sum of LTC4, LTD4, LTE4) showed no changes in either group studied. Statistically the results of cys-LTs in EBC were identical independent of units (measurement) used (ppm of PA or $\mathrm{pg} / \mathrm{mL}$ ).

The dose of inhaled steroid used by study patients and $\mathrm{FEV}_{1}$ values had no effect on magnitude of the response of the cys-LTs and its duration.

Negative correlation was founded between provocation doses of aspirin and exhaled $\mathrm{LTC}_{4}(r=-0.47, P=0.04)$, $\operatorname{LTD}_{4}(r=-0.46, P=0.04)$ and $\operatorname{LTE}_{4}(r=-0.43, P=0.05)$ levels only in AIA patients.

Exhaled Breath Condensate Concentration of Leukotriene $B_{4}$. At baseline (Table 2), exhaled breath condensate level of leukotriene $\mathrm{B}_{4}$ did not differ significantly between the AIA and ATA groups $(P=0.36)$.

Following aspirin administration, EBC levels of $\mathrm{LTB}_{4}$ decreased significantly only in aspirin-sensitive patients (ANOVA, $P=0.03$ ). No differences were observed when $\mathrm{LTB}_{4}$ was expressed as $\mathrm{pg} / \mathrm{mL}$.

Exhaled Breath Condensate Concentration of HETE. At baseline (Table 2), exhaled breath condensate levels of 5-HETE and 15-HETE were significantly higher in AIA as compared to ATA groups $(P=0.03, P=0.001$, resp.). Figure 1 . Following bronchial aspirin administration, EBC levels of 5and 15-HETE (ANOVA, $P=0.23$ ) remained unchanged in AIA ( $P=0.37$ and $P=0.23$, resp.) and ATA patients.

At baseline (Table 2), exhaled breath condensate levels of 12-HETE did not differ significantly between the AIA and ATA groups $(P=0.13)$. Following aspirin administration, EBC levels of 12-HETE decreased significantly only in ATA group $(P=0.03$, due to outliers Wilcoxon Matched pairs test was used). 


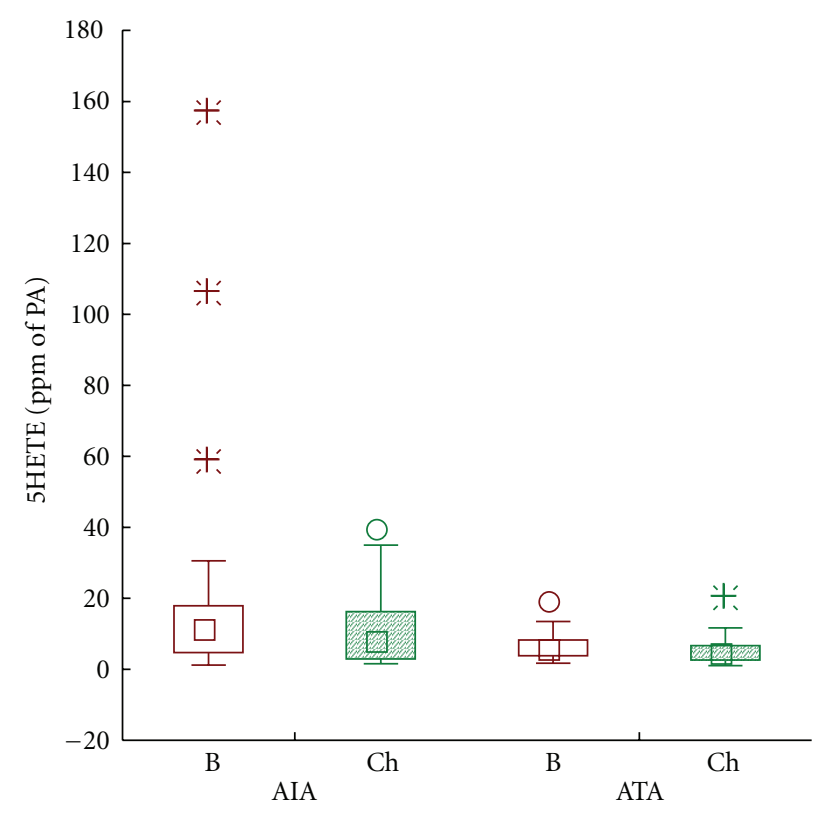

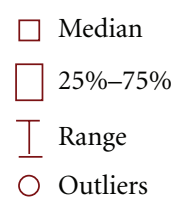

\begin{abstract}
米 Extremes
B Baseline

Ch Challenge
\end{abstract}

O Outliers
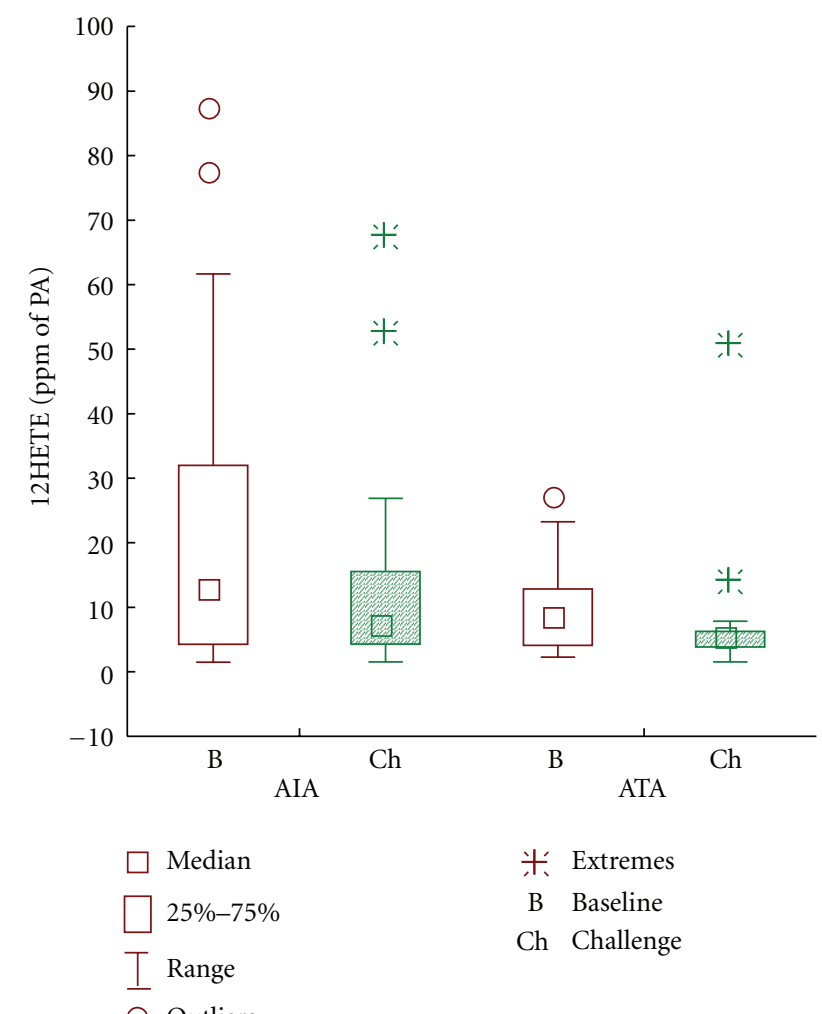

米 Extremes

B Baseline

Ch Challenge

(a)

(b)

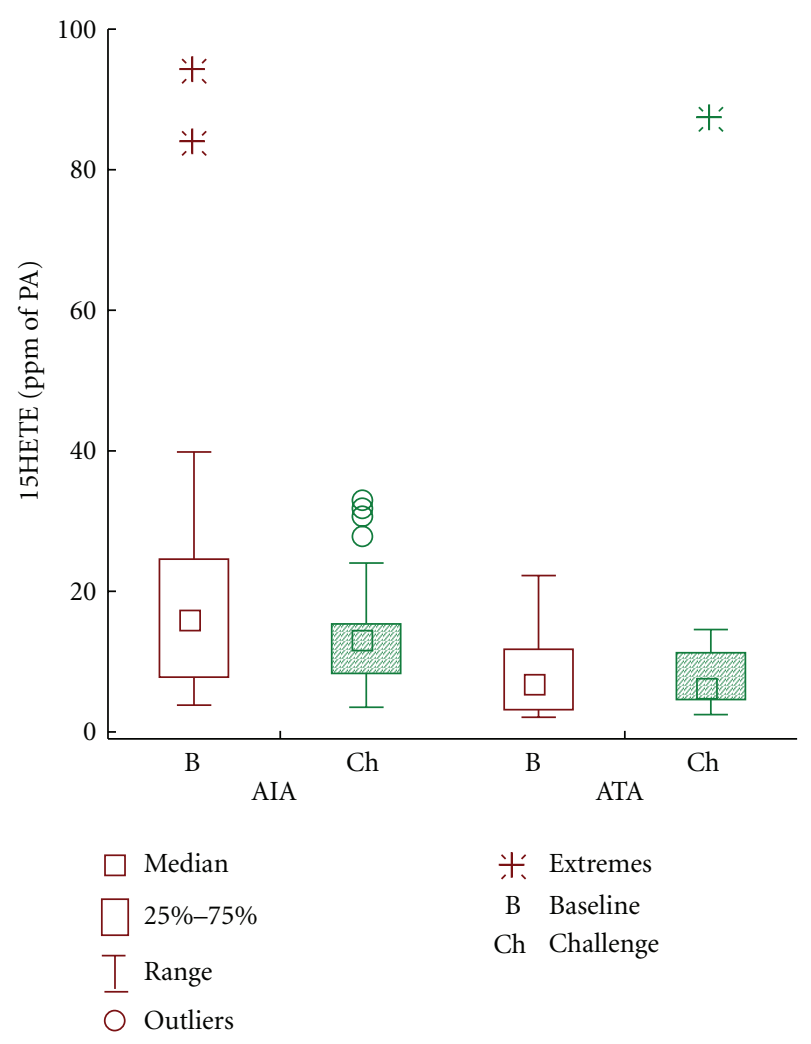

(c)

FIGURE 1: Eicosanoids' levels in exhaled breath condensate before and after bronchial aspirin challenge. (a) 5-HETE, (b) 15-HETE, and (c) 12-HETA. AIA: aspirin-induced asthma. ATA: aspirin-tolerant asthma. B: baseline. Ch: challenge. 
TABLE 3: Eicosanoids values at baseline and following aspirin challenge in AIA and ATA patients. Results of eicosanoids were recalculated as picograms per milliliter $(\mathrm{pg} / \mathrm{mL})$.

\begin{tabular}{|c|c|c|c|c|c|}
\hline & \multicolumn{2}{|c|}{$\operatorname{AIA}(n=21)$} & \multicolumn{2}{|c|}{$\operatorname{ATA}(n=23)$} & \multirow{2}{*}{$\begin{array}{c}P \text { (ANOVA) } \\
\text { After the challenge }\end{array}$} \\
\hline & Baseline & After the challenge & Baseline & After the challenge & \\
\hline $\mathrm{PGD}_{2}$ & $1.49 \pm 1.17$ & $1.54 \pm 1.02$ & $2.35 \pm 2.06$ & $1.80 \pm 1.22$ & \\
\hline$(\mathrm{pg} / \mathrm{mL})$ & 1.01 & 1.21 & 1.64 & 1.53 & 0.35 \\
\hline GC/MS & $(0.82 \div 1,68)$ & $(0.79 \div 2.11)$ & $(1.09 \div 2.70)$ & $(0.85 \div 3.20)$ & \\
\hline $\mathrm{PGD}_{2}$ & $1.53 \pm 0.81$ & $1.14 \pm 0.58$ & $1.76 \pm 1.14$ & $1.63 \pm 0.93$ & \\
\hline$(\mathrm{pg} / \mathrm{mL})$ & 1.39 & 1.09 & 1.48 & 1.45 & 0.05 \\
\hline HPLC & $(0.93 \div 2.02)$ & $(0.67 \div 1.36)$ & $(0.99 \div 2.19)$ & $(0.80 \div 2.07)$ & \\
\hline $9 \alpha 11 \beta \mathrm{PGF}_{2}$ & $0.48 \pm 0.33$ & $0.50 \pm 0.34$ & $0.32 \pm 0.18$ & $0.31 \pm 0.17$ & \\
\hline$(\mathrm{pg} / \mathrm{mL})$ & 0.36 & 0.34 & 0.29 & 0.28 & 0.59 \\
\hline GC/MS & $(0.16 \div 0.69)$ & $(0.25 \div 0.75)$ & $(0.20 \div 0.35)$ & $(0.21 \div 0.38)$ & \\
\hline PGF $2 \alpha$ & $0.42 \pm 0.34$ & $0.40 \pm 0.35$ & $0.43 \pm 0.33$ & $0.39 \pm 0.45$ & \\
\hline$(\mathrm{pg} / \mathrm{mL})$ & 0.28 & 0.27 & 0.30 & 0.23 & 0.82 \\
\hline GC/MS & $(0.19 \div 0.56)$ & $(0.15 \div 0.59)$ & $(0.23 \div 0.44)$ & $(0.16 \div 0.35)$ & \\
\hline 6-keto-PGF1 $\alpha$ & $8.94 \pm 4.30$ & $8.70 \pm 3.72$ & $9.95 \pm 4.54$ & $9.55 \pm 4.19$ & \\
\hline$(\mathrm{pg} / \mathrm{mL})$ & 7.17 & 7.22 & 7.48 & 7.46 & 0.53 \\
\hline GC/MS & $(6.75 \div 7.79)$ & $(7.02 \div 7.62)$ & $(7.24 \div 15.02)$ & $(7.24 \div 11.49)$ & \\
\hline 6-keto-PGF1 $\alpha$ & $8.99 \pm 4.41$ & $8.59 \pm 3.93$ & $9.69 \pm 4.37$ & $9.46 \pm 3.95$ & \\
\hline$(\mathrm{pg} / \mathrm{mL})$ & 7.26 & 6.96 & 7.40 & 7.65 & 0.60 \\
\hline HPLC/MS/MS & $(6.32 \div 8.41)$ & $(6.59 \div 8.54)$ & $(7.08 \div 13.54)$ & $(7.13 \div 10.27)$ & \\
\hline 11-dehydro TXB2 & $5.74 \pm 0.87^{*}$ & $5.94 \pm 0.89$ & $6.52 \pm 0.92 *$ & $6.39 \pm 0.94$ & \\
\hline$(\mathrm{pg} / \mathrm{mL})$ & 5.74 & 6.17 & 6.81 & 6.62 & 0.18 \\
\hline GC/MS & $(5.14 \div 6.34)$ & $(5.16 \div 6.49)$ & $(6.35 \div 7.00)$ & $(5.70 \div 7.16)$ & \\
\hline 11-dehydro TXB2 & $5.51 \pm 0.90^{*}$ & $5.96 \pm 1.11$ & $6.40 \pm 1.17^{*}$ & $6.18 \pm 1.01$ & \\
\hline$(\mathrm{pg} / \mathrm{mL})$ & 5.49 & 6.03 & 6.63 & 6.18 & $0.03^{*}$ \\
\hline HPLC/MS/MS & $(4.77 \div 6.16)$ & $(5.24 \div 6.55)$ & $(6.01 \div 7.03)$ & $(5.55 \div 7.09)$ & \\
\hline $\mathrm{LTC}_{4}$ & $4.17 \pm 4.56$ & $3.69 \pm 7.01$ & $4.35 \pm 4.57$ & $1.87 \pm 1.73^{*}$ & \\
\hline$(\mathrm{pg} / \mathrm{mL})$ & 2.30 & 2.02 & 2.46 & 1.06 & $0.01^{*}$ \\
\hline HPLC/MS/MS & $(1.23 \div 6.42)$ & $(1.13 \div 3.36)$ & $(1.05 \div 6.18)$ & $(0.59 \div 2.74)$ & \\
\hline $\mathrm{LTD}_{4}$ & $0.88 \pm 0.60$ & $0.85 \pm 0.59$ & $1.58 \pm 1.28$ & $1.18 \pm 0.88$ & \\
\hline$(\mathrm{pg} / \mathrm{mL})$ & 0.69 & 0.65 & 1.34 & 0.95 & 0.16 \\
\hline HPLC/MS/MS & $(0.43 \div 1.19)$ & $(0.50 \div 1.03)$ & $(0.55 \div 2.04)$ & $(0.41 \div 1.77)$ & \\
\hline $\mathrm{LTE}_{4}$ & $2.03 \pm 0.92$ & $3.20 \pm 3.63^{*}$ & $2.46 \pm 1.43$ & $2.78 \pm 1.44^{*}$ & \\
\hline$(\mathrm{pg} / \mathrm{mL})$ & 1.83 & 2.33 & 1.93 & 2.45 & $0.04^{*}$ \\
\hline HPLC/MS/MS & $(1.28 \div 2.71)$ & $(1.57 \div 3.44)$ & $(1.30 \div 3.45)$ & $(1.54 \div 3.69)$ & \\
\hline Total cysLTs & $7.30 \pm 4.98$ & $8.02 \pm 10.75$ & $8.39 \pm 5.62$ & $5.83 \pm 3.40$ & \\
\hline$(\mathrm{pg} / \mathrm{mL})$ & 5.97 & 6.01 & 7.24 & 4.90 & 0.24 \\
\hline HPLC/MS/MS & $(4.14 \div 9.66)$ & $(3.91 \div 6.75)$ & $(3.06 \div 12.75)$ & $(2.87 \div 8.87)$ & \\
\hline $\mathrm{LTB}_{4}$ & $69.09 \pm 102.42$ & $26.15 \pm 25.60^{*}$ & $54.23 \pm 99.10$ & $34.81 \pm 37.83$ & \\
\hline$(\mathrm{pg} / \mathrm{mL})$ & 29.66 & 16.99 & 27.73 & 15.35 & $0.02^{*}$ \\
\hline HPLC/MS/MS & $(10.26 \div 50.00)$ & $(10.41 \div 30.38)$ & $(6.40 \div 57.18)$ & $(6.37 \div 53.27)$ & \\
\hline 5 HETE & $7.15 \pm 9.80$ & $3.60 \pm 2.69$ & $2.75 \pm 1.94$ & $2.61 \pm 2.99$ & \\
\hline$(\mathrm{pg} / \mathrm{mL})$ & 2.94 & 3.41 & 2.29 & 1.67 & 0.79 \\
\hline HPLC/MS/MS & $(1.96 \div 7.41)$ & $(1.67 \div 5.29)$ & $(1.43 \div 3.12)$ & $(0.78 \div 3.00)$ & \\
\hline 12 HETE & $6.64 \pm 6.16$ & $5.85 \pm 10.45$ & $4.03 \pm 2.68$ & $3.45 \pm 6.75^{*}$ & \\
\hline$(\mathrm{pg} / \mathrm{mL})$ & 4.05 & 2.82 & 3.10 & 1.93 & $0.02 *$ \\
\hline HPLC/MS/MS & $(2.91 \div 7.76)$ & $(1.52 \div 4.22)$ & $(1.53 \div 5.84)$ & $(1.25 \div 2.85)$ & \\
\hline
\end{tabular}


TABle 3: Continued.

\begin{tabular}{|c|c|c|c|c|c|}
\hline & \multicolumn{2}{|c|}{$\operatorname{AIA}(n=21)$} & \multicolumn{2}{|c|}{$\operatorname{ATA}(n=23)$} & \multirow{2}{*}{$\begin{array}{c}P(\text { ANOVA }) \\
\text { After the challenge }\end{array}$} \\
\hline & Baseline & After the challenge & Baseline & After the challenge & \\
\hline 15 HETE & $6.99 \pm 4.87^{*}$ & $5.81 \pm 5.14$ & $3.48 \pm 2.78^{*}$ & $5.29 \pm 11.76$ & \\
\hline$(\mathrm{pg} / \mathrm{mL})$ & 5.96 & 4.77 & 2.95 & 2.39 & 0.30 \\
\hline HPLC/MS/MS & $(4.04 \div 8.27)$ & $(2.53 \div 6.52)$ & $(1.75 \div 3.98)$ & $(1.92 \div 2.97)$ & \\
\hline $\mathrm{EXC}_{4}$ & $1.05 \pm 0.82$ & $0.92 \pm 0.89$ & $0.89 \pm 0.57$ & $0.83 \pm 0.73$ & \\
\hline$(\mathrm{pg} / \mathrm{mL})$ & 0.79 & 0.59 & 0.80 & 0.61 & 0.74 \\
\hline HPLC/MS/MS & $(0.45 \div 1.61)$ & $(1.18 \div 0.58)$ & $(0.36 \div 1.40)$ & $(0.25 \div 1.07)$ & \\
\hline $\mathrm{EXD}_{4}$ & $1.71 \pm 2.29$ & $1.78 \pm 2.82$ & $1.70 \pm 1.76$ & $1.29 \pm 1.54$ & \\
\hline$(\mathrm{pg} / \mathrm{mL})$ & 0.73 & 0.62 & 1.06 & 0.74 & 0.909 \\
\hline HPLC/MS/MS & $(0.30 \div 1.51)$ & $(0.24 \div 1.93)$ & $(0.27 \div 2.31)$ & $(0.39 \div 1.96)$ & \\
\hline $\mathrm{EXE}_{4}$ & $5.47 \pm 8.61$ & $3.68 \pm 6.01$ & $4.01 \pm 4.97$ & $2.58 \pm 2.63$ & \\
\hline$(\mathrm{pg} / \mathrm{mL})$ & 2.15 & 1.05 & 1.59 & 1.35 & 0.07 \\
\hline HPLC/MS/MS & $(0.97 \div 4.60)$ & $(0.71 \div 2.66)$ & $(0.89 \div 5.66)$ & $(0.72 \div 3.89)$ & \\
\hline 8 -iso-PGF2 $\alpha$ & $0.25 \pm 0.12^{*}$ & $0.28 \pm 0.21$ & $0.33 \pm 0.15^{*}$ & $0.32 \pm 0.10$ & \\
\hline$(\mathrm{pg} / \mathrm{mL})$ & 0.20 & 0.21 & 0.35 & 0.34 & 0.92 \\
\hline GC/MS & $(0.17 \div 0.28)$ & $(0.19 \div 0.26)$ & $(0.20 \div 0.42)$ & $(0.25 \div 0.39)$ & \\
\hline
\end{tabular}

Median (25\% and $75 \%$ percentiles).

AIA: aspirin-induced asthma. ATA: aspirin-tolerant asthma.

${ }^{*} P$-values $<0.05$; AIA versus ATA at baseline or after the challenge.

$P$-values:

* AIA versus ATA at baseline.

* baseline versus challenge in AIA.

*baseline versus challenge in ATA.

The dose of inhaled steroid used by patients and $\mathrm{FEV}_{1}$ values had no effect on magnitude of the response of the 5and 15-HETE. At baseline, negative correlation was found between the doses of steroids and EBC levels of 12-HETE only in aspirin-sensitive subjects $(r=-0.45, P=0.04)$.

The dose of aspirin, had no effect on the magnitude of response of 5- and 15-HETE.

Exhaled Breath Condensate Concentration of Eoxins. At baseline and following aspirin challenge, exhaled breath condensate levels of eoxins $\mathrm{C}_{4}, \mathrm{D}_{4}$, and $\mathrm{E}_{4}$ did not differ significantly between the AIA and ATA groups (see Table 2).

At baseline and following aspirin challenge no correlation was found between provocation doses of aspirin, inhaled steroid therapy and $\mathrm{FEV}_{1}$ values, and eoxins EBC levels in aspirin-sensitive and aspirin-intolerant patients.

\section{Discussion}

In this study, we used a validated analytic platform [27, 30, 31] to analyze eicosanoids in EBC of asthmatic patients. A highly sensitive method of gas chromatography/mass spectrometry or high-performance liquid chromatography/mass spectrometry or both were used to measure spectrum of eicosanoids-nonvolatile compounds present in $\mathrm{EBC}[3,27$, 31]. Novel concepts for the standardization of EBC material measurements have been introduced to obtain characteristics of eicosanoid patterns produced by asthmatic lungs [27]. The assessment of palmitic acid content in EBC among many other methods [32] seems to be a convenient solution for compensating the "dilution factor" [3]. It has recently been demonstrated that dilution of nonvolatile compounds in water differs between the subjects by more than 1 order of magnitude and depends on ventilation mechanics $[33,34]$. For that reason, in this paper, data are recalculated as parts per million of palmitic acid. For better understanding, EBC eicosanoid levels are given in both applicable units: ppm of PA (Table 2) and in pg/mL (Table 3) independently of performed assay.

The aim of this study was to compare a wide profile of eicosanoids released to the epithelial surface of the asthmatic lung in subjects with and without aspirin hypersensitivity at baseline and following bronchial aspirin challenge, most of them on chronic inhaled steroid therapy. We demonstrated for the first time exhaled eicosanoids following local administration of aspirin in aspirin-induced asthma patients.

Comparing subjects with AIA and ATA no significant differences were observed in EBC levels of cyclooxygenase pathway prostanoid products $\left(\mathrm{PGD}_{2}\right.$ and its metabolite $9 \alpha 11 \beta \mathrm{PGF}_{2}, \mathrm{PGF} 2 \alpha, 6$-keto-PGF1 $\alpha$, and 11-dehydro $\mathrm{TXB}_{2}$ ) and nonenzymatic isomer (8-iso- $\left.\mathrm{PGF}_{2}\right)$ in neither baseline nor after aspirin inhalation. Opposing data has been earlier reported as lower [28] and other times higher [27] baseline $\mathrm{PGD}_{2}$ metabolite, namely, $9 \alpha, 11 \beta \mathrm{PGF}_{2}$, levels in EBC in AIA patients. Differences observed in this previous study between both asthma phenotypes [27] can possibly be explained by lower $\mathrm{FEV}_{1}$ values and more severe of disease in patients with aspirin hypersensitivity compared to subjects who tolerated aspirin well. Moreover, a significant predictor of decreased $\mathrm{FEV}_{1}$ was increased $9 \alpha, 11 \beta-\mathrm{PGF}_{2}$ only in AIA which did 
not correlate in ATA subjects [27]. In our study, all patients presented comparable $\mathrm{FEV}_{1}$ values, and most likely, similar bronchoconstricting eicosanoid levels such as $\mathrm{PGD}_{2}$ and its metabolite. Mast cells are probably the main source of $\mathrm{PGD}_{2}$ overproduction. Higher global production of $\mathrm{PGD}_{2}$ metabolite $9 \alpha, 11 \beta \mathrm{PGF}_{2}$ was also present in the blood and urine at baseline in AIA subjects $[18,20]$. Concentration of $9 \alpha, 11 \beta-\mathrm{PGF}_{2}$ was not changed by the oral-systemic [28] and how indicated our data bronchial-local administration of aspirin.

Our results revealed a significant upregulation of some arachidonate lipoxygenation products in asthmatic subjects with aspirin hypersensitivity, as manifested by high baseline levels of 5-, 15-HETE in EBC. This data is consistent with the latest observations [27]. These findings are related to an overexpression of lipoxygenases enzymes, particularly 5- and 15-LO in the asthmatic lung with aspirin hypersensitivity. These enzymes are expressed in eosinophils, activated macrophages, and also in lymphocytes and mast cells. Kowalski and colleagues have demonstrated that aspirin triggers specific generation of 15-HETE from nasal polyp epithelial cells [12] and peripheral blood leukocytes [35, 36] from aspirin-sensitive but not aspirin-tolerant subjects with asthma/rhinosinusitis. Also, they have demonstrated that two alternatively spliced variants of COX-1 mRNA present in human leucocytes may be differently expressed in patients with asthma. The relative expression of those variants has been correlated to aspirin-triggered 15-HETE generation suggesting association of this phenomenon with the pathogenesis of aspirin-induced asthma [37].

No elevation of baseline 12-HETE in EBC comparing AIA and ATA subjects was observed. Whereas, after bronchial aspirin administration, there was a statistically significant decrease in 12-HETE concentration noted only in ATA subjects. On that basis, we assume blood platelets, the main source of 12-LO, may possibly play some role in pathogenesis of aspirin hypersensitivity. However, 12-LO was originally cloned from respiratory epithelia, where 15-LO activity was also found [38].

Our study did not demonstrated baseline local overproduction of cys-LTs in the airways in AIA and is consistent with an earlier study [28]. Baseline EBC levels of LTC $_{4}$, $\mathrm{LTD}_{4}$, and $\mathrm{LTE}_{4}$ did not differ between aspirin tolerant and hypersensitive subjects. Contrary to this finding in subjects with AIA, a higher baseline level of cys-LTs in EBC was also reported $[26,27]$. This might have been a result of low $\mathrm{FEV}_{1}$ values and minor control of asthma (severity index) [27] or steroid-naive [26] in aspirin hypersensitivities compared to aspirin tolerant subjects. The levels of exhaled cys-LTs were lower in those AIA subjects who received inhaled steroid therapy [26]. Following bronchial aspirin challenge levels of particular cys-LTs showed some variations but the total concentration of cys-LTs remained unchanged in both study groups. Up till now, cys-LTs level in EBC has not been measured after local (inhaled) aspirin administration. However, it has been shown [28] that cys-LTs levels in EBC after oral (systemic administration) aspirin challenge increased significantly in subjects with AIA. Varying results of different studies implicate that levels of cys-LTs in EBC cannot be a convenient indicator of asthma phenotype as their level after challenge test possibly depends on manner of aspirin administration and probably the doses of steroids inhaled. Results can be surprising because, a key enzymeLTC4 synthase, is overexpressed in bronchial mucosa of patient with AIA [39]. Furthermore, circulating steroid blood eosinophils-main source of $\mathrm{LTE}_{4}$ - carry more mRNA transcripts for this enzyme [40].

As was reported in childhood asthma [41] and adult asthmatics [27], 15-LO analogues of cys-LTs, eoxins $\mathrm{C}_{4}, \mathrm{D}_{4}$, and $\mathrm{E}_{4}$, showed no increase at baseline in asthmatic subjects independent of aspirin hypersensitivity. Their concentrations were not changed by the bronchial aspirin challenge. Role of those eicosanoids has been recently investigated in aspirin hypersensitive patients.

The high-sensitivity eicosanoid profiling of lipoxygenation products (5HETE, 15HETE) in EBC makes it possible to detect alterations in asthma, especially in its distinct phenotype characterized by hypersensitivity to aspirin and other nonsteroidal anti-inflammatory drugs. Cysteinyl-leukotriene levels in EBC after aspirin challenge in stable asthma patients, not steroid naive, most probably cannot be used as a reliable and sensitive index for aspirin hypersensitivity. In stable AIA patients on chronic inhaled steroid therapy of global (urinary) rather than in local (breath condensate) production of postchallenge cys-LTs is of greater and more sensitive value for aspirin hypersensitivity. We believe that quantitate cell analysis and measurements of released eicosanoides in induced-sputum will be more applicable for that purpose.

\section{List of Abbreviations}

$\begin{array}{ll}\text { AIA: } & \text { Aspirin-intolerant asthma } \\ \text { ATA: } & \text { Aspirin-tolerant asthma } \\ \text { EBC: } & \text { Exhaled breath condensate } \\ \text { GC-MS: } & \begin{array}{l}\text { Gas chromatography-mass } \\ \text { spectrometry }\end{array} \\ \text { HPLC-MS/MS: } & \begin{array}{l}\text { High-performance liquid } \\ \text { chromatography-tandem mass }\end{array} \\ & \text { spectrometry } \\ \text { COX: } & \text { Cyclooxygenase } \\ \text { PG: } & \text { Prostaglandin } \\ \text { 9 } \alpha, 11 \beta-\mathrm{PGF}_{2}: & \text { PGD } 2 \text { semistable metabolite } \\ \text { 6-keto-PGF } \alpha: & \text { Prostacyclin metabolite } \\ \text { 11-dehydro-TXB2: } & \text { Thromboxane A2 metabolite } \\ \text { EP2: } & \text { Prostaglandin receptor 2 } \\ \text { LO: } & \text { Lipoxygenase } \\ \text { HETE: } & \text { Hydroxyeicosatetraenoic acid } \\ \text { LT: } & \text { Leukotriene } \\ \text { Cys-LTs: } & \text { Cysteinyl leukotrienes } \\ \text { 8-iso-PGF } \alpha: & \text { Isoprostane F } \alpha \\ \text { NSAIDs: } & \text { Nonsteroidal anti-inflammatory drugs. }\end{array}$

\section{Acknowledgment}

This work was supported by the Grant K/PBW/0000552 from the Polish Ministry of Science. 


\section{References}

[1] I. Horváth, J. Hunt, P. J. Barnes et al., "ATS/ERS task force on exhaled breath condensate: methodological recommendations and unresolved questions," European Respiratory Journal, vol. 26, no. 3, pp. 523-548, 2005.

[2] P. Montuschi, "Exhaled breath condensate: 8-isoprostane and eicosanoids," in European Respiratory Monograph: Exhaled Biomarkers, I. Horvath and J. C. de Jongste, Eds., pp. 196-206, ERS, 2010.

[3] M. Sanak, A. Gielicz, K. Nagraba, M. Kaszuba, J. Kumik, and A. Szczeklik, "Targeted eicosanoids lipidomics of exhaled breath condensate in healthy subjects," Journal of Chromatography B: Analytical Technologies in the Biomedical and Life Sciences, vol. 878, no. 21, pp. 1796-1800, 2010.

[4] A. Szczeklik, E. Niżankowska-Mogilnicka, and M. Sanak, "Hypersensitivity to aspirin and nonsteroidal anti-inflammatory drugs," in Middleton's Allergy, N. F. Adkinson Jr., W. W. Busse, B. S. Bochner, S. T. Holgate, R. Lemanske, and F. E. R. Simons, Eds., pp. 1227-1243, Mosby, St. Louis, Mo, USA, 7th edition, 2009.

[5] A. Szczeklik, "The cyclooxygenase theory of aspirin-induced asthma," European Respiratory Journal, vol. 3, no. 5, pp. 588593,1990

[6] A. Szczeklik and M. Sanak, "The broken balance in aspirin hypersensitivity," European Journal of Pharmacology, vol. 533, no. 1-3, pp. 145-155, 2006.

[7] S. E. Wenzel, "Asthma: defining of the persistent adult phenotypes," Lancet, vol. 368, no. 9537, pp. 804-813, 2006.

[8] N. Higashi, H. Mita, E. Ono et al., "Profile of eicosanoid generation in aspirin-intolerant asthma and anaphylaxis assessed by new biomarkers," Journal of Allergy and Clinical Immunology, vol. 125, no. 5, pp. 1084-1091, 2010.

[9] P. E. Christie, P. Tagari, A. W. Ford-Hutchinson et al., "Urinary leukotriene $\mathrm{E}_{4}$ concentrations increase after aspirin challenge in aspirin-sensitive asthmatic subjects," American Review of Respiratory Disease, vol. 143, no. 5 I, pp. 1025-1029, 1991.

[10] M. Kumlin, B. Dahlén, T. Björck, O. Zetterström, E. Granström, and S.-E. Dahlén, "Urinary excretion of leukotriene $\mathrm{E}_{4}$ and 11-dehydro-thromboxane $\mathrm{B}_{2}$ in response to bronchial provocations with allergen, aspirin, leukotriene $\mathrm{D}_{4}$, and histamine in asthmatics," American Review of Respiratory Disease, vol. 146, no. 1, pp. 96-103, 1992.

[11] D. Schäfer, M. Schmid, U. C. Göde, and H. W. Baenkler, "Dynamics of eicosanoids in peripheral blood cells during bronchial provocation in aspirin-intolerant asthmatics," European Respiratory Journal, vol. 13, no. 3, pp. 638-646, 1999.

[12] M. L. Kowalski, R. Pawlicźak, J. Wozniak et al., "Differential metabolism of arachidonic acid in nasal polyp epithelial cells cultured from aspirin-sensitive and aspirin-tolerant patients," American Journal of Respiratory and Critical Care Medicine, vol. 161, no. 2 I, pp. 391-398, 2000.

[13] C. A. Pérez-Novo, J. B. Watelet, C. Claeys, P. van Cauwenberge, and C. Bachert, "Prostaglandin, leukotriene, and lipoxin balance in chronic rhinosinusitis with and without nasal polyposis," Journal of Allergy and Clinical Immunology, vol. 115, no. 6, pp. 1189-1196, 2005.

[14] M. Pierzchalska, Z. Szabo, M. Sanak, J. Soja, and A. Szczeklik, "Deficient prostaglandin E2 production by bronchial fibroblasts of asthmatic patients, with special reference to aspirininduced asthma," Journal of Allergy and Clinical Immunology, vol. 111, no. 5, pp. 1041-1048, 2003.

[15] S. Ying, Q. Meng, G. Scadding, A. Parikh, C. J. Corrigan, and T. H. Lee, "Aspirin sensitive rhinosinusitis is associated with reduce E-prostanoid 2 (EP2) receptor expression on nasal mucosal inflammatory cells," Journal of Allergy and Clinical Immunology, vol. 117, no. 2, pp. 312-318, 2006.

[16] N. Jinnai, T. Sakagami, T. Sekigawa et al., "Polymorphisms in the prostaglandin $\mathrm{E}_{2}$ receptor subtype 2 gene confer susceptibility to aspirin-intolerant asthma: a candidate gene approach," Human Molecular Genetics, vol. 13, no. 24, pp. 3203 3217, 2004.

[17] L. Mastalerz, M. Sanak, A. Gawlewicz-Mroczka et al., "Prostaglandin $\mathrm{E}_{2}$ systemic production in patients with asthma with and without aspirin hypersensitivity," Thorax, vol. 63, no. 1, pp. 27-34, 2008.

[18] A. Szczeklik, R. Dworski, L. Mastalerz et al., "Salmeterol prevents aspirin-induced attacks of asthma and interferes with eicosanoid metabolism," American Journal of Respiratory and Critical Care Medicine, vol. 158, no. 4, pp. 1168-1172, 1998.

[19] F. Gaber, K. Daham, A. Higashi et al., "Increased levels of cysteinyl-leukotrienes in saliva, induced sputum, urine and blood from patients with aspirin-intolerant asthma," Thorax, vol. 63, no. 12, pp. 1076-1082, 2008.

[20] G. Bochenek, K. Nagraba, E. Nizankowska, and A. Szczeklik, "A controlled study of $9 \alpha 1 \beta-\mathrm{PGF}_{2}$ (a prostaglandin $\mathrm{D}_{2}$ metabolite) in plasma and urine of patients with bronchial asthma and healthy controls after aspirin challenge," Journal of Allergy and Clinical Immunology, vol. 111, no. 4, pp. 743-749, 2003.

[21] N. Higashi, M. Taniguchi, H. Mita, M. Osame, and K. Akiyama, "A comparative study of eicosanoid concentrations in sputum and urine in patients with aspirin-intolerant asthma," Clinical and Experimental Allergy, vol. 32, no. 10, pp. 1484-1490, 2002.

[22] N. R. Ferreri, W. C. Howland, D. D. Stevenson, and H. L. Spiegelberg, "Release of leukotrienes, prostaglandins, and histamine into nasal secretions of aspirin-sensitive asthmatics during reaction to aspirin," American Review of Respiratory Disease, vol. 137, no. 4, pp. 847-854, 1988.

[23] M. L. Kowalski, "Aspirin-sensitive rhinosinusitis and asthma," Clinical Allergy and Immunology, vol. 19, pp. 147-175, 2007.

[24] A. Szczeklik, K. Sladek, R. Dworski et al., "Bronchial aspirin challenge causes specific eicosanoid response in aspirinsensitive asthmatics," American Journal of Respiratory and Critical Care Medicine, vol. 154, no. 6, pp. 1608-1614, 1996.

[25] L. Mastalerz, M. Sanak, A. Gawlewicz, A. Gielicz, J. Faber, and A. Szczeklik, "Different eicosanoid profile of the hypersensitivity reactions triggered by aspirin and celecoxib in a patient with sinusitis, asthma, and urticaria," Journal of Allergy and Clinical Immunology, vol. 118, no. 4, pp. 957-958, 2006.

[26] A. Antczak, P. Montuschi, S. Kharitonov, P. Gorski, and P. J. Barnes, "Increased exhaled cysteinyl-leukotrienes and 8-isoprostane in aspirin-induced asthma," American Journal of Respiratory and Critical Care Medicine, vol. 166, no. 3, pp. 301306, 2002.

[27] M. Sanak, A. Gielicz, G. Bochenek et al., "Targeted eicosanoid lipidomics of exhaled breath condensate provide a distinct pattern in the aspirin-intolerant asthma phenotype," Journal of Allergy and Clinical Immunology, vol. 127, pp. 1141-1147, 2011.

[28] M. Sanak, B. Kiełbasa, G. Bochenek, and A. Szczeklik, "Exhaled eicosanoids following oral aspirin challenge in asthmatic patients," Clinical and Experimental Allergy, vol. 34, no. 12, pp. 1899-1904, 2004.

[29] E. Niżankowska-Mogilnicka, G. Bochenek, L. Mastalerz et al., "EAACI/GA2LEN guideline: aspirin provocation tests for diagnosis of aspirin hypersensitivity," Allergy, vol. 62, no. 10, pp. 1111-1118, 2007. 
[30] R. C. Murphy, R. M. Barkley, K. Z. Berry et al., "Electrospray ionization and tandem mass spectrometry of eicosanoids," Analytical Biochemistry, vol. 346, no. 1, pp. 1-42, 2005.

[31] D. Tsikas, "Application of gas chromatography-mass spectrometry and gas chromatography-tandem mass spectrometry to assess in vivo synthesis of prostaglandins, thromboxane, leukotrienes, isoprostanes and related compounds in humans," Journal of Chromatography B: Biomedical Sciences and Applications, vol. 717, pp. 201-245, 1998.

[32] H. Dressel, F. Muller, R. Fischer et al., "Independentinformation of nonspecific biomarkers in exhaled breath condensate," Respiration, vol. 80, pp. 401-409, 2010.

[33] J. B. McCafferty, T. A. Bradshaw, S. Tate, A. P. Greening, and J. A. Innes, "Effects of breathing pattern and inspired air conditions on breath condensate volume, $\mathrm{pH}$, nitrite, and protein concentrations," Thorax, vol. 59, no. 8, pp. 694-698, 2004.

[34] K. Schwarz, H. Biller, H. Windt, W. Koch, and J. M. Hohlfeld, "Characterization of exhaled particles from the healthy human lung-a systematic analysis in relation to pulmonary function variables," Journal of Aerosol Medicine and Pulmonary Drug Delivery, vol. 23, no. 6, pp. 371-379, 2010.

[35] M. L. Kowalski, A. Ptasinska, M. Jedrzejczak et al., "Aspirintriggered 15-HETE generation in peripheral blood leukocytes is a specific and sensitive aspirin-sensitive patients identification test (ASPITest)," Allergy, vol. 60, no. 9, pp. 1139-1145, 2005.

[36] M. L. Kowalski, A. Ptasińska, B. Bienkiewicz, R. Pawliczak, and L. DuBuske, "Differential effects of aspirin and misoprostol on 15-hydroxyeicosatetraenoic acid generation by leukocytes from aspirin-sensitive asthmatic patients," Journal of Allergy and Clinical Immunology, vol. 112, no. 3, pp. 505-512, 2003.

[37] M. L. Kowalski, M. Borowiec, M. Kurowski, and R. Pawliczak, "Alternative splicing of cyclooxygenase-1 gene: altered expression in leucocytes from patients with bronchial asthma and association with aspirin-induced 15-HETE release," Allergy, vol. 62, no. 6, pp. 628-634, 2007.

[38] A. R. Brash, W. E. Boeglin, and M. S. Chang, "Discovery of a second 15S-lipoxygenase in humans," Proceedings of the National Academy of Sciences of the United States of America, vol. 94, no. 12, pp. 6148-6152, 1997.

[39] M. Sanak, M. Pierzchalska, S. Bazan-Socha, and A. Szczeklik, "Enhanced expression of the leukotriene $\mathrm{C}_{4}$ synthase due to overactive transcription of an allelic variant associated with aspirin-intolerant asthma," American Journal of Respiratory Cell and Molecular Biology, vol. 23, no. 3, pp. 290-296, 2000.

[40] C. Jenkins, J. Costello, and L. Hodge, "Systematic review of prevalence of aspirin induced asthma and its implications for clinical practice," British Medical Journal, vol. 328, no. 7437, pp. 434-437, 2004.

[41] C. Sachs-Olsen, M. Sanak, A. M. Lang et al., "Eoxins: a new inflammatory pathway in childhood asthma," Journal of Allergy and Clinical Immunology, vol. 126, no. 4, pp. 859-867, 2010. 


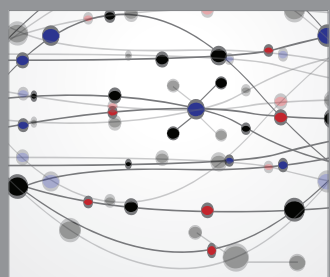

The Scientific World Journal
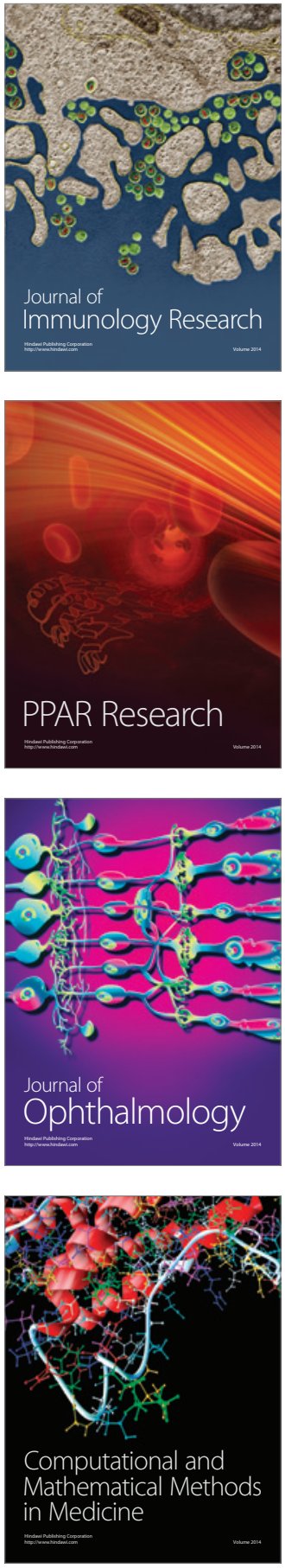

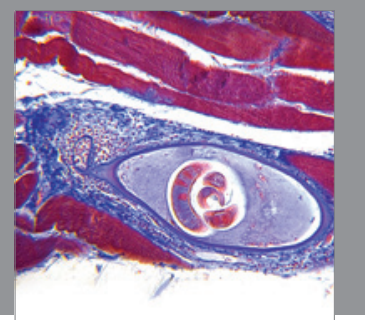

Gastroenterology

Research and Practice
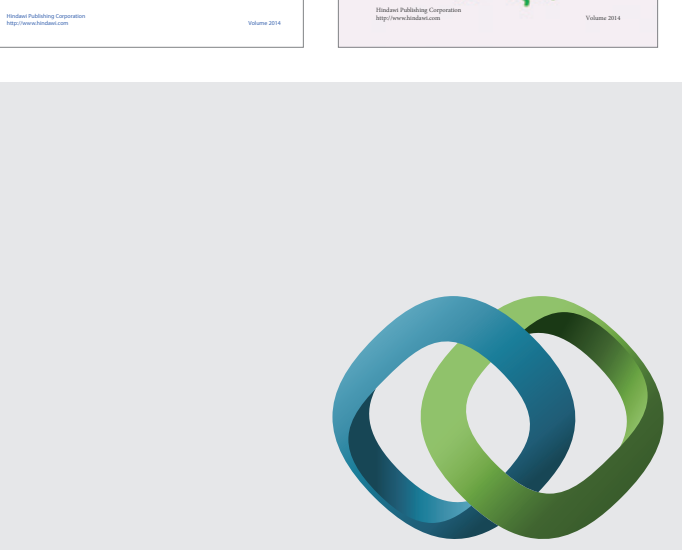

\section{Hindawi}

Submit your manuscripts at

http://www.hindawi.com
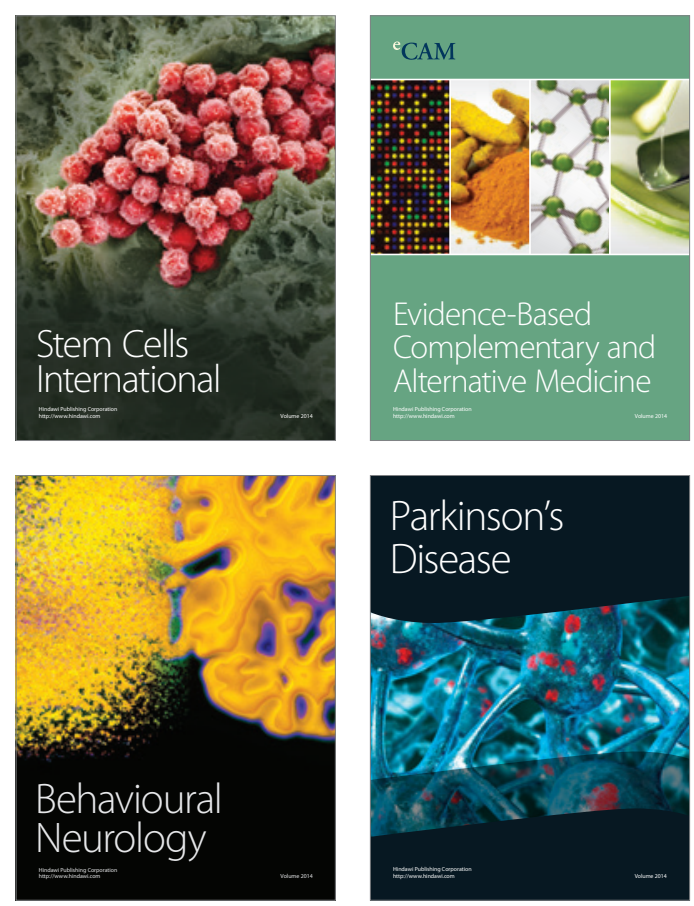

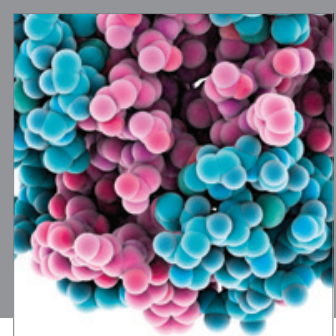

Journal of
Diabetes Research

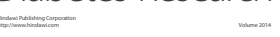

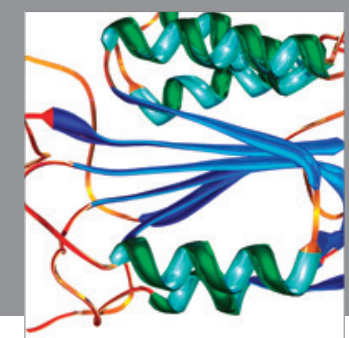

Disease Markers
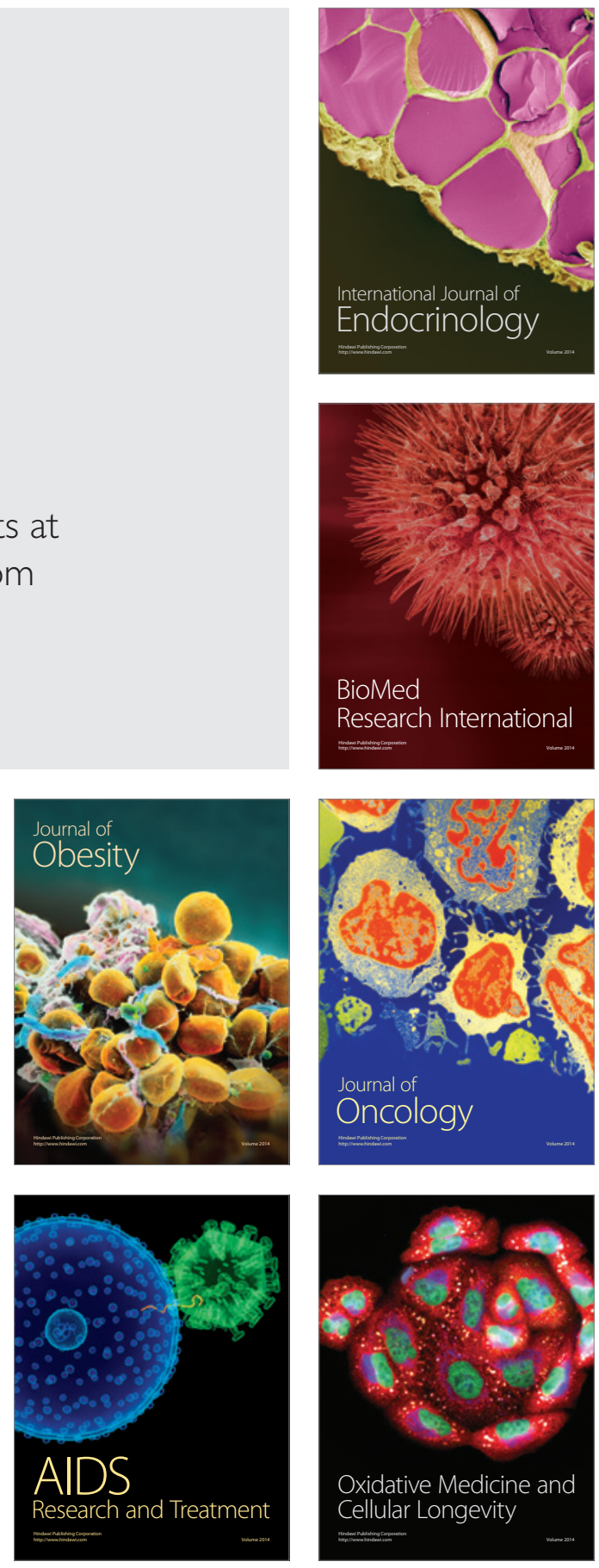